\section{$\S 17$. Liquid Lithium Divertor System for Fusion Reactor}

Nagayama, Y.

One of the biggest technical issues in the steady state fusion reactor is the heat load onto the divertor wall. A 3 GW reactor makes the alpha heating power of $600 \mathrm{MW}$. The alpha heating power heats the plasma and is finally removed by the radiation and by the diverter. Suppose the major radius is $10 \mathrm{~m}$, the divertor leg thickness is $1 \mathrm{~cm}$ and the radiation loss is negligibly small, the heat flux is 500 $\mathrm{MW} / \mathrm{m}^{2}$. Different designs and different conditions provide slightly different results, but the heat flux is in the order of $100 \mathrm{MW} / \mathrm{m}^{2}$. This is far beyond the power handling capability of any solid material in steady state. If the radiation removes $99 \%$ of the alpha heating power, this problem may be solved, but the plasma confinement should be deteriorated.

This paper proposes another solution that is a liquid lithium divertor system, as shown in Fig. 1. The proposed divertor system has an evaporation chamber and a differential pumping chamber. In the evaporation chamber, the liquid lithium is hold by a wick, which is mesh or capillary, as a target. The strong surface tension allows installing the liquid lithium on the ceiling of the evaporation chamber. Vaporization of lithium removes the heat coming from the fusion plasma along the divertor leg. Since the latent heat of lithium is $2.273 \times 10^{7}[\mathrm{~J} / \mathrm{kg}], 26 \mathrm{~kg}$ or 50 liters of lithium is evaporated every second to remove the heat of 600 MW. Large amount of liquid lithium should be circulated in order to keep the wick wet always.

The lithium vapor is condensed on the wall of the evaporation chamber. The coolant of the wall transfers the heat to the power generator. Since lithium and water violently reacts, water cannot be a good coolant of the wall. Possible coolant is gas and it requires large area because of poor cooling efficiency. The wall area can be large enough to optimize the heat transfer.

The differential pumping chamber is installed between the evaporation chamber and the plasma chamber in order to prevent the lithium vapor leakage. The high heat flux is due to the narrow divertor leg, of which width is about $1 \mathrm{~cm}$. By using this, narrow slits can be installed to connect divertor chambers, and the conductance of the lithium vapor can be significantly reduced. The lithium vapor is pumped down in the pumping chamber. Thus leakage of lithium vapor can be minimized. Since many experiments have reported that the lithium wall gives good effect to the plasma confinement, leakage of lithium vapor may be allowed $[1,2]$. By using this system, the divertor heat load problem in the steady state fusion reactor could be solved.

We have many unknown problems to make the liquid lithium diverter. Especially, the power handling capability of the wick and the circulation rate are unknown. The liquid lithium diverter system should be tested using a high heat flux sheet beam, which should be long and narrow.

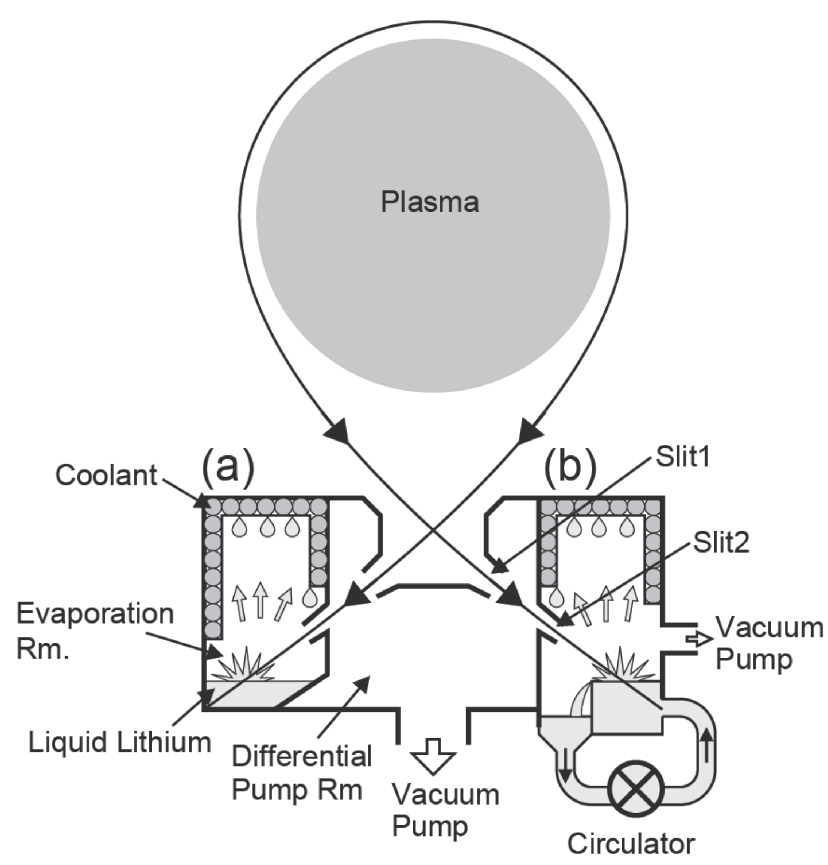

Fig.1 Liquid lithium diverter system. This system works both in tokamak and helical system.

[1] V.A. Evtikhin, et al., Fusion Eng. Des. 49-50, 195 (2000).

[2] R. Kaita, R. Majeski, et al., Fusion Eng. Des. 61-62, 217-222 (2002). 